\title{
An Analytical Model for Improvement of Linearity and Efficiency of RF Linear Power Amplifiers for Radar Systems
}

\author{
Amir Al-Mslmany, Member IACSIT, Caiyun Wang, and Qunsheng Cao
}

\begin{abstract}
Power amplifier is the key component in radar systems, so linearity and efficiency is the most important parameters in order to maintain the performance of power amplifier, based on matlab curve fitting toolbox an analytical model for improvement of linearity and efficiency of radio frequency (RF) linear power amplifiers (PAs) is introduced. This model simplifies the traditionally complicated methods of analysis of RF PA. According to a change of the direct current (DC) bias as a function of the alternative current (AC) input signal of RF linear PA the linearity and efficiency can be improved.
\end{abstract}

Index Terms - AC and DC analyses, ADS, biasing, efficiency linearity.

\section{INTRODUCTION}

Power amplifiers are the master component in wireless transceivers, especially in radar systems their function is to amplify the signal and generate the required RF power that allows transmission of the signal over the appropriate range.Linear power amplifiers are needed in many modern wireless communication systems, such as radar systems, enhanced data rate Bluetooth, wireless local area network. One of the issues that faced in design of linear amplifiers for specific digital modulation standards is how to simulate and predict the behaviour of the design when amplifying modulated signals, the stringent requirement of linearity complicates the design of transceivers [1], especially design of the power amplifier module in transmitters.

A recently works on PAs showed that the linearity can be optimized by tuning the source/load impedance of the baseband and the 2nd harmonic frequency [2]. The traditionally nonlinear analysis method is more difficult because it uses more complicated mathematical solutions, such as voltera series [3] based on small signal model transistors, while it hardly describes the large signal nonlinearity [4] for the omission of the DC offset with AC input. Wu Tuo Chen, Hongyi and Qian Dahong [5] introduced a model to improve the linearity of RF PAs; this model indicates that the DC bias resistance can affect the nonlinearity, but this model is very difficult to study, and more complicated to make any modification required for improvement of linearity and efficiency, this paper introduces a computer simulation analytic model to improve

Manuscript received November 25, 2013; revised January 30, 2014.

The authors are with Nanjing University of aeronautics and astronautics, college of electronic and information engineering, NANJING 210016 China (e-mail: mslmany@gmail.com, a.mslmany@nuaa.edu.cn, wangcaiyun@nuaa.edu.cn, qunsheng@nuaa.edu.cn). the linearity and efficiency of RF linear PAs.

\section{THEORETICAL ANALYSIS}

The circuit shown in Fig. 1 introduces the commonly used DC bias circuit for linear bipolar PAs (regular bias), $\left(I_{\text {Bias }}=\right.$ const, $\left.R_{\text {Bias }}=\infty\right)$.

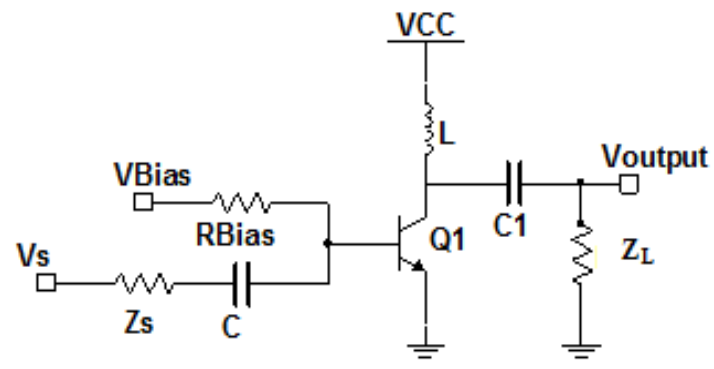

Fig. 1. Regular bias model.

Fig. 2 shows the simplified large signal equivalent circuit of a bipolar linear PA based on Gummel Boon (GB) model.

It consists of $C_{\text {couple }}$ (the DC blocking capacitor) and $L_{\text {choke }}$ ( the RF chock), $Z_{S}$ is the source impedance and $Z_{L}$ is the load impedance, $C_{d}$ is the base diffusion capacitance, which is related to the forward transmit time $\tau$ and the collector current $i_{c} . C_{j e}$ and $C_{j c}$ are the junction capacitances of $\mathrm{BE}$ and $\mathrm{BC}$ respectively, which are approximately constant compared to the nonlinear $C_{d}$.

The collector and base voltage-controlled current sources are respectively [5]:

$$
i_{c}=I_{S}\left(\exp \frac{V_{B E}+v_{b e}}{V_{T}}-1\right) \approx I_{o} \exp \frac{v_{b e}}{V_{T}}
$$

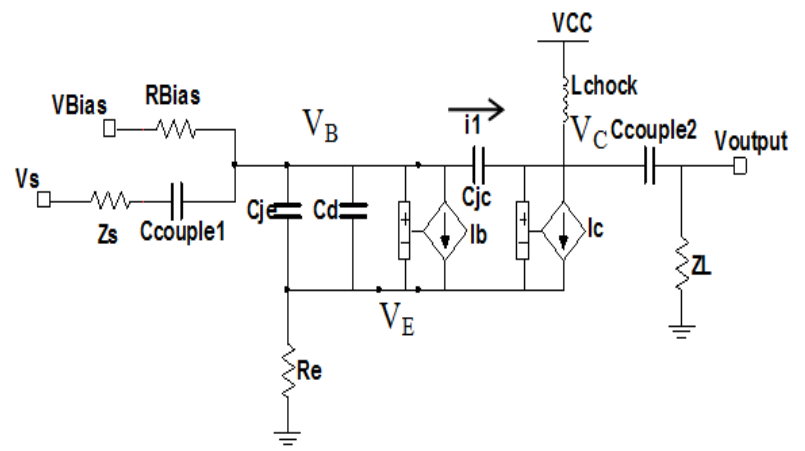

Fig. 2. Large signal equivalent circuit of a bipolar linear power amplifier.

$$
i_{b} \approx \frac{i_{c}}{\beta}=\frac{I_{o}}{\beta} \exp \frac{v_{b e}}{V_{T}}
$$

where

$$
I_{o}=I_{S} \exp \frac{V_{B E}}{V_{T}}
$$


Using the AC and DC analysis for the show circuits it can be easily to derive the following equations:

$$
I_{o}=\frac{I_{o q}}{1+\frac{K V_{s m}^{2}}{1+\frac{\beta V_{T}}{I_{o q} R_{\text {Bias }}}}}
$$

where

$$
K=\frac{\left|C_{1}(S)\right|^{2}}{4}
$$

is constant, $C_{1}(s)$ is solved by voltera series which is more difficult to solve it numerically, if the bias is an ideal current source, so $R_{\text {Bias }}=\infty$, and

$$
I_{o} \approx \frac{I_{o q}}{1+K V_{s m}^{2}} \approx I_{o q}\left(1-K V_{s m}^{2}\right)
$$

From equation (5) it is seen that $I_{\mathrm{o}}$ would reduce with an increase of $V_{s m}$ and $R_{\text {Bias }}$.

Draw equation (4) using matlab, and then using Matlab curve fitting toolbox to get:

$$
I_{o}=\frac{a_{i} * e^{b_{i} 10 \log R_{\text {Bias }}}}{C_{1}+d_{i} * e^{e_{i}^{*} \log R_{\text {Bias }}}} \times V_{\text {sm }}{ }^{2}+1
$$

where $a_{i}=-0.01296, b_{i}=2.103, \quad c_{i}=1.145, d_{i}=0.0621$, $e_{i}=2.106$ are the constants of curve fitting.

Fig. 3 shows the relation between (normalized) $I_{\mathrm{o}}$ and $\left(V_{s m}\right)^{2}$ at different $R_{\text {Bias }}$ for two cases:

- Previous model using matlab.

- Present model using matlab curve fittingtoolbox.

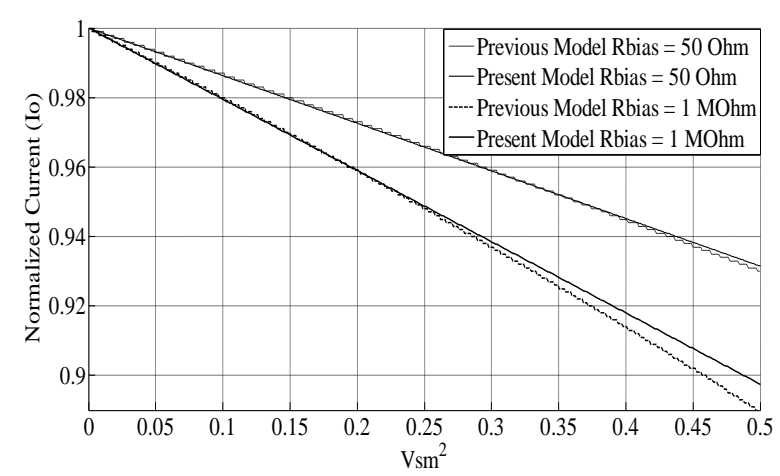

Fig. 3. Curves of the DC current $I_{\mathrm{o}}$ versus $V_{s m}$ and $R_{\text {Bias }}$.

Comparing these curves it is found that the error between the two curves is less than $2 \%$, thus equation (6) is approximately the same as equation (4).

Assume an input composed of two adjacent tones,

$$
\begin{aligned}
& V_{s}=\frac{V_{s m}^{2}}{\sqrt{2}}\left(\cos \omega_{1} t+\cos \omega_{2} t\right)=\frac{V_{s m}}{2 \sqrt{2}}\left(e^{s_{1} t}+e^{-s_{1} t}+\right. \\
& \quad e s 2 t+e-s 2 t
\end{aligned}
$$

The $3^{\text {rd }}$ intermodulation ratio is[6]:

$$
|I M R 3|=\left|\frac{3}{4} \frac{F_{3}\left(S_{1}, S_{1},-S_{2}\right)}{F_{1}\left(s_{1}\right)}\right|\left|\frac{V_{s m}}{\sqrt{2}}\right|^{2}=\frac{3\left|V_{s m}\right|^{2}}{8} f_{1}\left(I_{o}\right)
$$

where

$$
\left|C_{1}(S)\right|^{2} \mid \frac{E_{3}(S)}{E_{1}(S)}-C_{1}(S) B_{3}(S)+2 B_{2} C_{1}\left[C_{1}(S) B_{2}(S)-\right.
$$

and

$$
B_{2} C_{1}=\frac{2 B_{2}(0) C_{1}(0)+B_{2}(2 S) C_{1}(2 S)}{3}
$$

The function of $f_{1}\left(I_{o}\right)$ is complex but also it can be plotted using MATLAB and then to use Matlab Curve Fitting Toolbox to get the approximated form:

$$
\left.f_{1}\left(I_{o}\right)=a_{f} \times \mathrm{e}^{b_{f} \times I_{0}}+c_{f} \times e^{d_{f} \times I_{0}}\right)
$$

With constants $(a, b, c$ and $d)$ and its values are:

$$
\begin{array}{ll}
a_{f}=138.7, & b_{f}=-13.25, \\
c_{f}=14.34, & d_{f}=-2.823
\end{array}
$$

Fig. 4 shows the relation between the (normalized $f_{1}\left(I_{o}\right)$ and $\left(I_{\mathrm{o}}\right)$ for two different cases the same as Fig. 3.

Comparing these curves it is found that the error between the two curves is less than $2 \%$, thus equation (11) is approximately the same as equation (9).

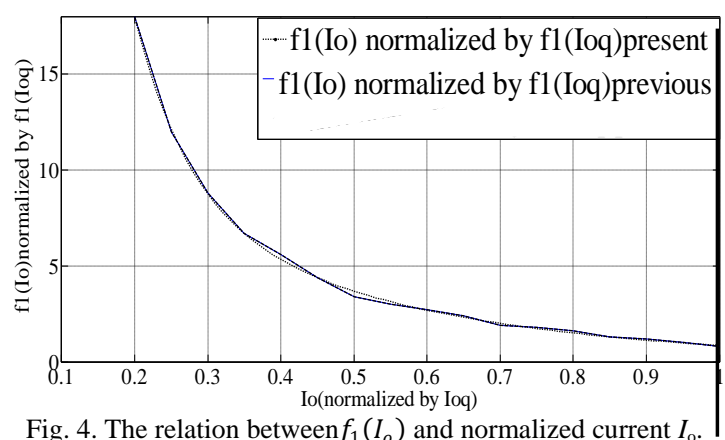

Fig. 4. The relation between $f_{1}\left(I_{o}\right)$ and normalized current $I_{\mathrm{o}}$.

From Fig. 3 and Fig. 4 it is seen that $f_{1}\left(I_{o}\right)$ will increase as $V_{s m}$ increases, so that the $3 \mathrm{rd}$ order intermodulation product (IMR3 ) would reduce compared to the standard results, which means the IMR3 is proportional to $\left|V_{s m}\right|^{2}$. Equation (6) indicates that a larger bias resistance would generate more nonlinearity. Thus, to gain a better linearity in the design of an RF linear PA, $R_{\text {Bias }}$ should be chosen to be small, so a diode bias model is introduced. Fig. 5 shows the DC bias circuit for linear bipolar PA (diode bias)

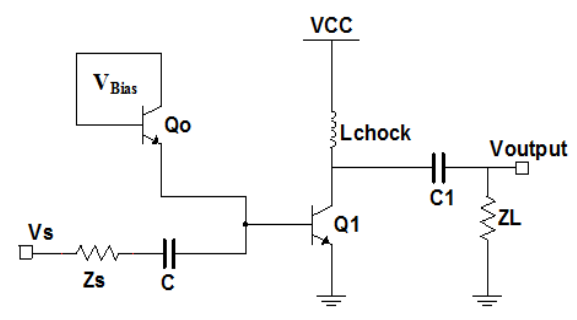

Fig. 5. Diode bias.

Generally, the size of the diode is very small, which means that the AC resistance of the bias branch can be neglected in 
the analysis of the linear PA.Using the same analysis as in the case of regular bias to get:

$$
I_{o} \approx I_{o q}\left(1-\frac{K V_{s m}^{2}}{2}\right)
$$

Comparing equation (12) and equation (5) we can see that the offset of $I_{\mathrm{o}}$ as a function of an increase in $V_{s m}$ for the diode biased PA equation (12) is approximately half that for a regular current biased PA equation (5), so the current $I_{\mathrm{o}}$ for the diode bias is greater than $I_{\mathrm{o}}$ for regular bias and hence the linearity of the diode biased PA is better than the regular bias [7].

From the comparative results of the two bias types, it was shown that the diode bias can improve the linearity by decreasing the drop of the DC current $I_{0}$, so if the bias can completely compensate the drop, the linearity can be further improved.

Fig. 6 shows the circuit of the proposed model for improving linearity of linear PA.

This model reduces the leakage from the bias branches, the bias resistance can be large $\left(R_{a d} ; R_{\text {Bias }} \gg>R_{e}\right)$, Using the same analysis as in the case of regular bias and diode bias we find that:

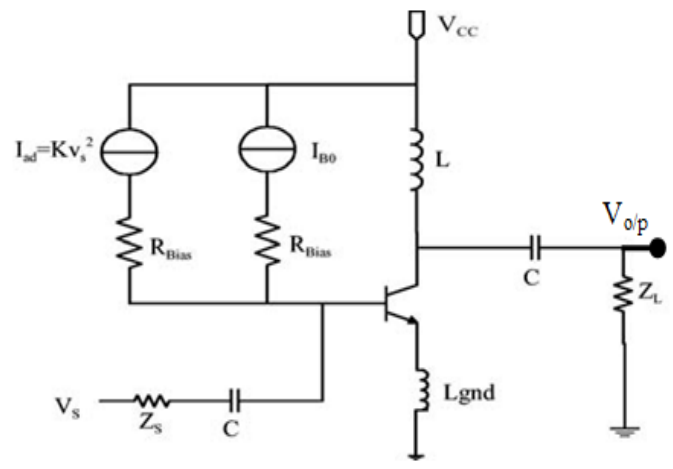

Fig. 6. The proposed bias model of RF linear PA.

This model reduces the leakage from the bias branches, the bias resistance can be large $\left(R_{a d} ; R_{\text {Bias }}>>R_{e}\right)$, Using the same analysis as in the case of regular bias and diode bias we find that:

$$
V_{T} \ln \frac{I_{O}}{I_{s}}+R_{\text {Bias }}\left(\frac{I_{o}+i_{c \mid D C}}{\beta}-i_{a d}\right)-V_{\text {Bias }}=0
$$

where

$$
i_{a d}=\frac{i_{c \mid D C}}{\beta}=\frac{I_{o}\left|\mathrm{C}_{1}(\mathrm{~S})\right|^{2}}{4 \beta} V_{s m}{ }^{2}
$$

The DC voltage $v_{b e}$ and the DC current $I_{\mathrm{o}}$ will not be affected by $V_{s m}$. So, the linearity could be improved.

\section{Simulation}

The circuit shown in Fig. 6 used for ADS simulation for the bias model of RF linear PA, the adaptive DC current bias $I_{a d}$ is proportional to the input AC power:

$$
I_{a d}=K_{1} v_{s}^{2}
$$

Using the harmonic balance (HB) simulation, with sweeping

$$
K_{1}: \text { From }(0) \text { to }\left(K_{o p t}=\frac{I_{B 0\left|C_{1}(S)\right|^{2}}}{4}\right)
$$

The output power is shown in Fig. 7. From this figure it is seen that the bias could improve the maximum linear output power.

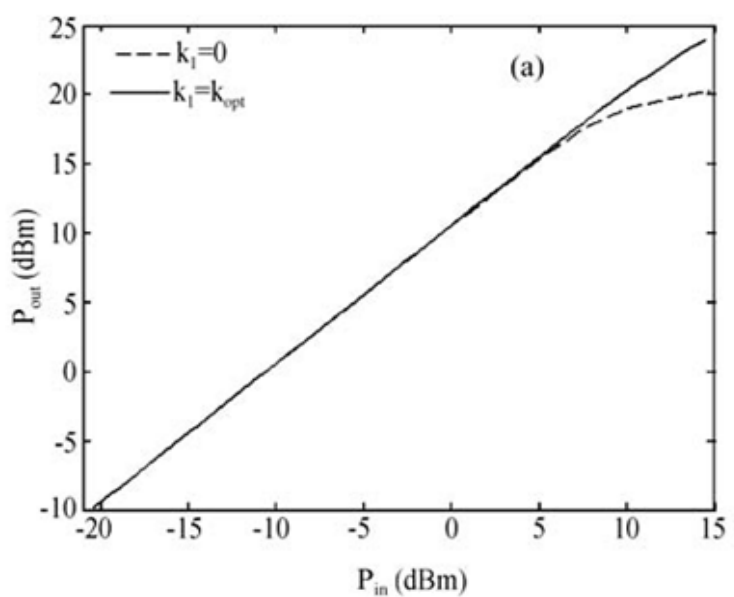

Fig. 7. Simulated output power.

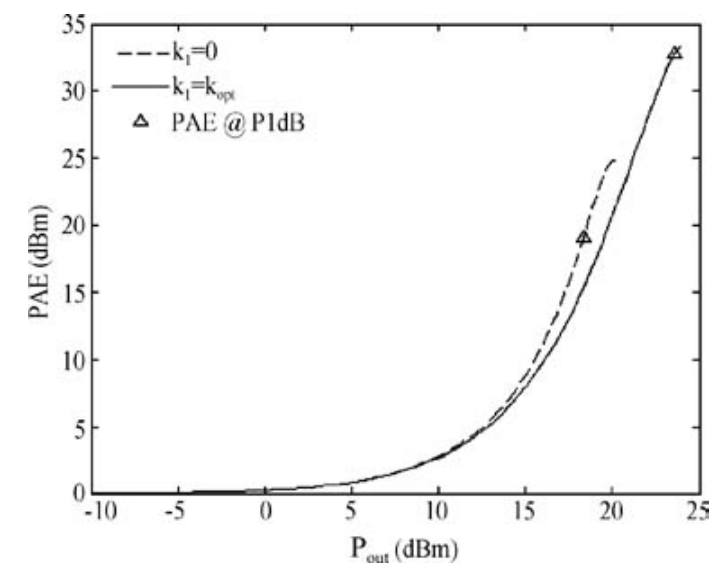

Fig. 8. Simulated power added efficiency of different bias PAs.

Fig. 8 shows that although the power added efficiency (PAE) of the improved bias PA is lower, the PAE at $P_{1 \mathrm{~dB}}$ can be increased from $20 \%$ to $32 \%$ when increasing $P_{1 \mathrm{~dB}}$.

\section{RESUlTS}

From the present simulation it is found that larger bias resistance would generate more nonlinearity.

Thus, to gain a better linearity in the design of an RF linear PA, $R_{\text {Bias }}$ should be chosen to be small.

\section{CONCLUSION}

In this paper an analytical model is proposed for simplicity of numerical analysis of the regular bias model to improve the linearity of linear PA. This analytical model goes away from the difficult mathematical problems, such as voltera series.

Also this paper discusses the effect of biasing on linearity and efficiency of the linear power amplifier.

\section{ACKNOWLEDGMENT}

I would like to thank the past and present Staff Members of 
the College of Electronic and Information Engineering of Nanjing University of Aeronautics and Astronautics for their great support and guidance especially my great respectable professor Qunsheng Cao. and Dr. Caiyun Wang.

\section{REFERENCES}

[1] A. Burr, Modulation and Coding for Wireless Communications, Beijing: Publishing House of Electronics Industry, 2003.

[2] J. Vuolevi and T. Rahkonen, "The effects of source impedance on the linearity of BJT common-emitter amplifiers," in Proc. the International Symposium on Circuits and Systems, vol. 4, 2000.

[3] S. C. Cripps, RF Power Amplifiers for Wireless Communications, Norwood, Artech House Inc, 1999.

[4] N. B. Carvalho and J. C. Pedro, "Large and small signal IMD behavior of microwave power amplifiers," IEEE Trans Microw Theory Tech, 1999, vol. 47, pp. 2364.

[5] T. C. Wu Y. Hong, and D. H. Qian, "Theoretical analysis and an improvement method of the bias effect on the linearity of RF linear power amplifiers," Institute of Microelectronics, vol. 30, no. 5, 2009.

[6] P Wambacq, Distortion Analysis of Analog Integrated Circuits, Kluwer Academic Publishers, 1998.

[7] S. N. Youn and S. P. Chul, "PCS/W-CDMA dual-band MMIC power amplifier with a newly proposed linearizing bias circuit," IEEE J Solid State Circuits, 2002, vol. 37, no. 1096.

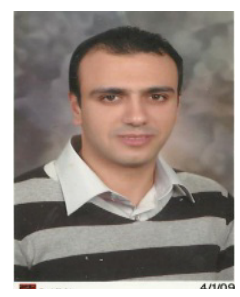

Amir Al-Mslmany was born in Alexandria, Egypt on 20 February, 1982. He received his B.S. in electronic engineering and the He received his M.S. in communications and electronics from faculty of engineering, Alexandria University, Alexandria, Egypt, in 2003. He is currently in Ph.D. degree in radar system in Nanjing University of Aeronautics and Astronautics, college of electronic and information engineering, Nanjing, China;

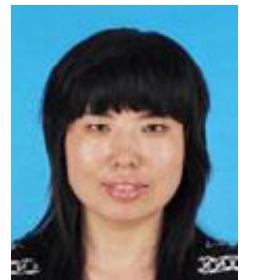

Caiyun Wang was born in Shanxi, China on September 30, 1975. She graduated in 1996 with a B.S. degree and in 1999 with a M.S. degree. She received the Ph.D. degree in signal and information processing from Beijing University, Beijing, China, in 2008. She is currently an associate professor with the School of Academy of Frontier Science, Nanjing University of Aeronautics and Astronautics (NUAA). Her major research interests are in the fields of radar automatic target recognition (RATA), radar signal processing, and adaptive signal processing, and pattern recognition.

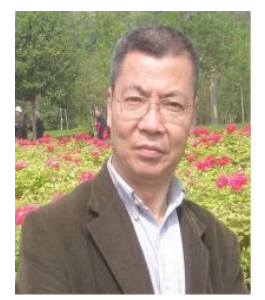

Qunsheng Cao was born in 1959. He received his Ph.D. in electrical engineering from The Hong Kong Polytechnic University in 2001. From 2001 to 2005 he worked as a research associate in the Department of Electrical Engineering, University of Illinois at Urbana-Champaign and at the Army High Performance Computing Research Center (AHPCRC), University of Minnesota. In 2005, Dr. Cao joined Nanjing University of Aeronautics and Astronautics (NUAA), China, as a professor of electrical engineering. Dr. Cao's current research interests are in computational electromagnetic, and particularly in time-domain numerical techniques (FDTD, MRTD and TDFEM) for the study of microwave devices and scattering applications. Dr. Cao has published more than thirty papers in refereed journals and conference proceedings and he is a co-author of the book multiresolution time domain scheme for electromagnetic engineering (series in microwave and optical engineering, vol. 1, Wiley, 2005). 